\title{
feel the pull of space
}

Gravitational-wave researchers have recently teamed up with high-energy physicists to explore how the world's largest particle accelerators could be used to detect the tiny ripples in space caused by gravitational waves. At a recent brainstorming meeting at CERN, researchers from both sides discussed whether the interaction between gravitational waves and accelerating subatomic particles could provide a new way of detecting the waves (www.cern.ch/Physics/ Conferences/C1999/Thinkshop).

Theorists predict that gravitational waves are emitted by astrophysical sources, such as binary stars and supernova explosions. The waves have so far escaped detection because their effect on the curvature of space-time is minuscule, typically just $l$ part in $10^{21}$. Several experiments are currently being built that will be sensitive enough to measure such changes. The idea behind these experiments is to look for variations in the distance between two masses located at the ends of the arms in a laser interferometer (see Physics World May $1999 \mathrm{p} 20$ ).

But gravitational waves could also deflect bunches of particles as they race around an accelerator. The idea has generated a great deal of interest among gravitational-wave researchers according to Jan van Holten, head of theoretical physics at the NIKHEF laboratory in the Netherlands.

It is no mean feat to accelerate subatomic particles to relativistic speeds around a $27 \mathrm{~km}$ storage ring, like the LEP collider at CERN. Over 500 so-called beam-position monitors are located around the accelerator to pinpoint the location of each bunch of particles. In principle, these detectors could be used to measure the bean deflection due to a gravitational wave. The waves will also alter the frequency with which the particles race àround the ring, a change that could be measured more accurately. The effect should be most pronounced in the biggest accelerators suggests Daniel Zer-Zion from CERN.

But this does not spell the end for gravitational-wave interferometers. Particle accelerators are five or six orders of magnitude less sensitive than interferometers to the effects of low-frequency gravitational waves notes van Holten. And current beamposition monitors are not sensitive enough. However, the outlook for accelerators is much brighter for gravitational waves in the

: terahertz range that may have been produced in the early universe.

Phrsics Woncd aueust 1999 